\title{
Traffic Sign Recognition with Color-based Method, Shape-arc Estimation and SVM
}

\author{
David Soendoro $^{\# 1}$, Iping Supriana ${ }^{\# 2}$ \\ ${ }^{\#}$ School of Electrical Engineering and Informatics, Institut Teknologi Bandung \\ Ganesha 10 Bandung, Indonesia \\ ${ }^{1}$ if17086estudents.if.itb.ac.id \\ ${ }^{2}$ ipingeinformatika.org
}

\begin{abstract}
Traffic sign recognition is studied as one of the modern assistance in driving. The main purpose of it is to help driver on realizing traffic sign around the car by using computer vision enhanced to the car. In this paper, a combination method of Color-based Method and SVM is presented to do the traffic sign recognition. Color-based Method with CIELab + hue is chosen because it gives good result on localizing traffic signs. It is first used to preprocess the image to binary image. The binary image is then processed with canny to give more accurate result on detecting traffic signs. To get the traffic sign shape, the preprocessed image is checked by using Ramer-Douglas-Peucker algorithm, this algorithm will approximate each closed object shape in the preprocessed image. Detecting closed object make it unable to detect occluded and attached road signs. In order to detect occluded and attached signs, the proposed method will use two phase of detection by estimating the shape of the arc or later called the shape-arc algorithm. Approximated circle, square, or triangle images will be marked as traffic sign and processed in the recognition step with linear c-SVM. SVM classification will be based on binary images which gives $97 \%$ accuracy. The proposed method is more accurate than methods proposed by the reference papers and the detection is able to detect harder problems such as attached signs.
\end{abstract}

Keywords- Color-based method, CIELab, traffic sign recognition, shape arc estimation, Ramer-Douglas-Peucker, binary image, $S V M$.

\section{INTRODUCTION}

Accidents on the road is placed nineteen of the biggest cause of death in the world. In fact, accidents on road have killed more than diabetes mellitus and some kind of cancers including breast cancer and liver cancer. [10][11] Start from this awareness, intelligent traffic system is needed. Traffic signs hold important information to the driver but in high speed or bad road condition drivers sometimes cannot pay full attention on the traffic sign. Here is where traffic sign recognition system will have important role. By using camera, the car itself will be able to record all traffic signs that have been passed. Recognition will also make the driver more convenient on reading the traffic sign because sometimes driver do not know the meaning of the traffic sign especially when they go abroad. However, we still do not have perfect detection or recognition for traffic signs. The main problems are the rotating invariance and illumination such as weather and daytime.
In order to get rid of illumination problem the color space used must not be RGB. On RGB, the same thing can be captured as different color in a different condition. The relevant color space to make a robust method on gaining the color is the CIELab and HSV. The detail of CIELab will be described later. For the rotation invariance problem shape approximation can be used in detecting shape while binary image data for SVM parameter used for recognition.

On this paper, the detection will be made to upgrade the accuracy of detecting from Escalera's method by using HSV color space [4] and reducing computation cost from Fleyeh's method by avoiding the calculation of Euclidian distances [6]. The detection is an improvement from Fleyeh's Thesis [7] for detaching attached signs with shape arc counting for circle signs. Recognition will be almost the same with one used for Fleyeh's Thesis because it gives a very good result on both speed and accuracy. Other papers that was used as a comparison for this papers are [1], [2], [3], [5] are all using the color and shape recognition for detecting traffic and road signs. Many methods was presented but the best result is likely the Escalera's method[4] with HSV for detection and Fleyeh's method with IHLS[6] and SVM for recognition from Fleyeh's thesis [7].

The remaining parts of this paper are organized as follows: in section 2, the Color-based method, Ramer-Douglas-Peucker algorithm, shape arc detection, and binary images in SVM are described and in section 3 experiments on traffic signs recognition are presented followed by some conclusion in section 4.

\section{THEORY AND ALGORITHM}

\section{A. Color-based Method}

First step in the traffic sign recognition is to detect the position of the traffic sign. One of the fastest image detection methods is to threshold image's color. The most common threshold is to threshold the RGB component of each pixel. However, the main problem of RGB threshold is illumination. RGB threshold cannot differ between day and night. To get rid of this problem, CIELab color space is used. CIELab color space is a color-opponent space with dimension L for lightness and $\mathrm{a}$ and $\mathrm{b}$ for the color-opponent dimensions, based on nonlinearly compressed CIE XYZ color space coordinates. 
Unlike the RGB color models, Lab color is designed to approximate human vision. It aspires to perceptual uniformity, and its L component closely matches human perception of lightness. It can thus be used to make accurate color balance corrections by modifying output curves in the $a$ and $b$ components, or to adjust the lightness contrast using the $\mathrm{L}$ component

CIELab color space, as RGB color space, is built by 3 components. First is $\mathrm{L}^{*}$ for lightness, lightness gives information about the amount of light affecting the pixel. Second is $\mathrm{a}^{*}, \mathrm{a}^{*}$ represents redness - greenness. Third or the last is $b^{*}, b^{*}$ represents yellowness - blueness. For more details of the formula to convert RGB to CIELab can be read on the guide book of color, the Colorimetry. [9]

CIELab alone is not sufficient to differentiate many traffic signs clearly, therefore to improve the clarity of the threshold result image, hue as a representation of all color in 360 degrees is used. By using, $a^{*}, b^{*}$, and hue component we can differentiate traffic signs color from background color by making threshold of specific values. From color-based threshold, binary image is made. Transfer functions used are as mentioned below in equation 3 to 6 . Transfer functions are for the hue, $a^{*}$ and $b^{*}$.

$$
B_{r}=\left\{\begin{array}{lc}
0 & a^{*}<140 \text { and }(h \geq 20 \text { and } h \leq 340) \\
255 & a^{*} \geq 140 \text { and }(h<20 \text { or } h>340)
\end{array}\right.
$$

Equation 1 is for red images.

$$
B_{g}=\left\{\begin{array}{cc}
0 & a^{*} \geq 115 \text { and }(h \leq 110 \text { or } h \geq 150) \\
255 & a^{*}<115 \text { and }(h>110 \text { and } h<150)
\end{array}\right.
$$

Equation 2 is for green images.

$$
B_{y}=\left\{\begin{array}{cc}
0 & b^{*}<140 \text { and }(h \leq 30 \text { or } h \geq 70) \\
255 & b^{*} \geq 140 \text { and }(h>30 \text { and } h<70)
\end{array}\right.
$$

Equation 3 is for yellow images.

$$
B_{b}=\left\{\begin{array}{cc}
0 & b^{*} \geq 115 \text { and }(h \leq 180 \text { or } h \geq 260) \\
255 & b^{*}<115 \text { and }(h>180 \text { and } h<260)
\end{array}\right.
$$

Equation 4 is for blue images.

After image filtering with equation 1 to 4 , to obtain the edges, Gaussian blur and canny algorithm can be used. To improve detection accuracy and eliminating noises, it is recommended to use image dilatation and erosion.

\section{B. Ramer-Douglas-Peucker}

Taking binary image preprocessed before, Ramer-DouglasPeucker algorithm can be used to approximate the shape of the traffic sign. However, Ramer-Douglas-Peucker algorithm needs a list of points as its parameter. To get image's edges, it is needed to apply edge detection algorithm (on this paper canny edge detection is used).

The main idea of Ramer-Douglas-Peucker algorithm consists of these steps:

1. Take one base edge. (The one with the most extreme $\mathrm{X}$ or $\mathrm{Y}$ value - A).

2. Take farthest edge from $A(N)$.
3. Make line from A to $\mathrm{N}$.

4. Take farthest orthogonal distance edge from line AN (M).

5. Is $\mathrm{M}$ to $\mathrm{AN}>$ epsilon?

a. If true, make to lines $\mathrm{AM}$ and $\mathrm{MN}$, repeat to 1 .

b. Else, all edges can be simplified to line AN.

Ramer-Douglas-Peucker algorithm can be illustrated as figure 1 .

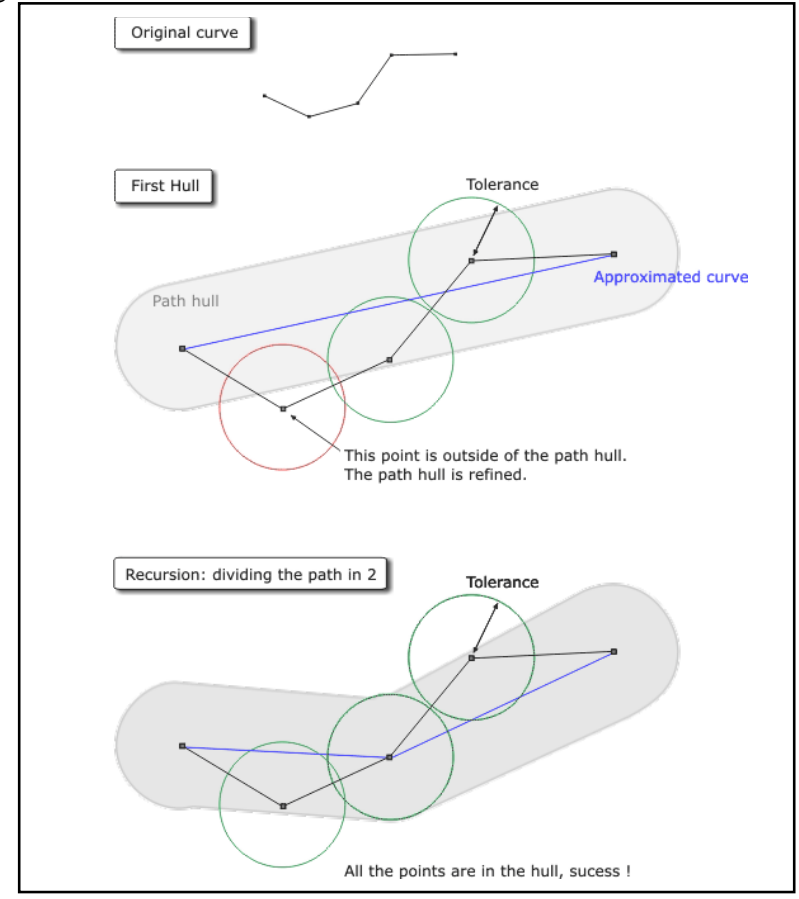

Figure 1. Ramer-Douglas-Peucker Algorithm

More details of the Ramer-Douglas-Peucker is available on the "Speeding Up the Douglas-Peucker LineSimplification Algorithm" by John Hershberger. [8]

From the simplified lists of edges we can approximate the shape of all polygons. On current implementation, the first approximation for circle or ellipse is based on fitEllipse2 function from OpenCV. However, for triangle, rectangular, and second phase approximation for circle detection, shapearc detection will be used.

\section{Shape-arc detection}

Shape-arc detection is an algorithm inspires by the real calculation of a simple object. For example is a circle or a rectangle.Shape-arc detection consists of three major algorithm. They are:

1. Arc extraction from contours

2. Arc grouping

3. Shape estimation

The first algorithm is the extraction from contours to arc. The contours that will be extracted is the one that has been detected from previous step (Ramer-Douglas-Peucker algorithm). An arc can be formed from three points. So the main idea of using the contour is to take for each three points 
which belongs to the same contour to make an arc. Do this to all the detected contours to generate a complete arcs of the image. However, make a list of complete arcs can be expensive. Smaller computational cost can be achieved by filtering the arc's magnitude. For instance, for circle only arcs with angle between 100 to 170 degrees is taken or rectangulars can only be formed by arcs with angle between 80 to 100 degrees.

Next step is to group the arcs into one object. In this step many algorithm can be used depend on the shape we want to detect. In the implementation, currently only circle, rectangle, and triangle have been implemented. First is circle, in a circle we can find the center of the circle by using the arc. For explanation, we can see it in figure 2 .

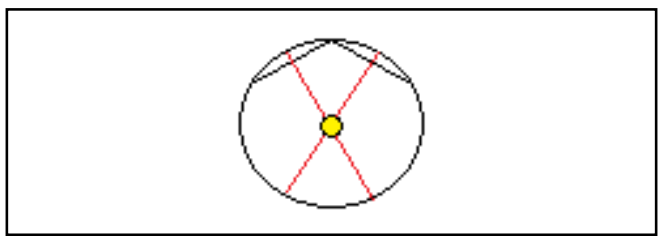

Figure 2. Every arc can has its own estimated circle

By making an upright line from a side of the arc in a circle, the estimated center can be found. A place where both upright line make an intersection is the center of the circle. With this center of the circle, then we can estimate the radius by calculating the distance from the center of the circle to the arc points. This is usable on the grouping step. In order on making a group, arcs which its estimated circle have close center and radius value are having higher possibility of being a group on forming a circle. The grouping step can be difference depends on the shape we want to detect. As for rectangle and triangle, the currently implemented algorithm is to make sure its angle is between 80 and 100 for rectangles and 50 to 70 for triangles and the total of the angles are around 360 for rectangles and 180 for triangles.

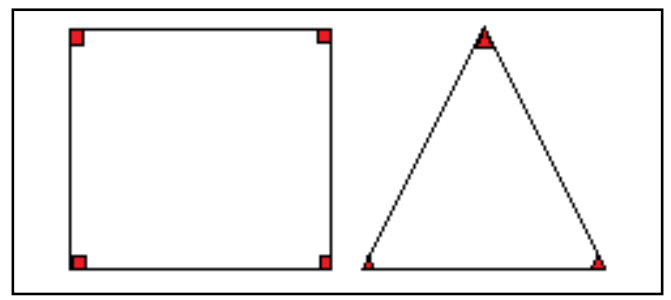

Figure 3. To group a rectangle or triangle, currently only by counting its angles

The main focus is the circle because most of traffic signs are of the shape of circle. Furthermore, this algorithm has successfully improve Fleyeh's thesis [7] on detaching attached signs. The experiment will be shown on next section.

After arc grouping, the last is the shape estimation. Shape estimation can be combined with arc grouping like for rectangle or triangle. But can also be divided. In case of detecting circle, all of the grouped arcs will be checked by calculating the degree they represent on the estimated circle. As in figure 4, arc a is forming the part of circle from 0 to 45 degree and arc $b$ is forming the part of circle from 45 to 90 degree. All the forming arcs will be counted from the agreed center of the arcs group.

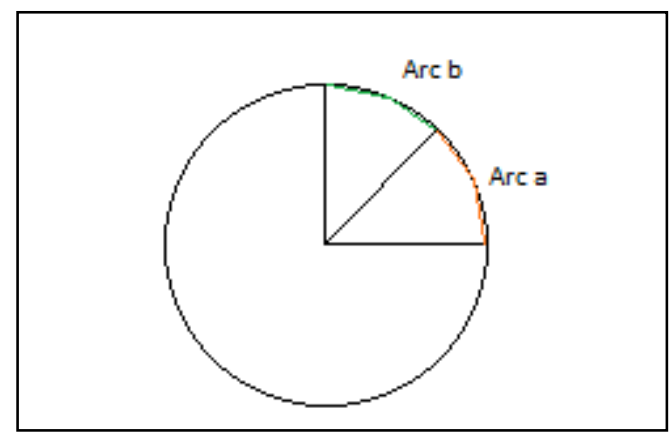

Figure 4. Shape estimation by counting all members of a grouped arcs

Then, by dividing the accomplished circle by 360 - total of a circle degree - the circle probability can be counted However, arc extraction from an image can be expensive. To make it faster, arc extraction can be the second phase of shape detection. In the implementation for instance, the shape-arc algorithm is used on detected ellipses by using the fitEllipse2 algorithm from OpenCV. The shape-arc detection algorithm is used to detached attached signs which result can be seen on the experiment section.

\section{Binary Images and SVM}

To classify traffic sign detected from the image, we can use a machine learning algorithm. On this paper, SVM is used because of its speed and good support with Binary images data. The SVM used is C-SVM with linear kernel. This is used because it gives very high accuracy and simple (97\%). The main idea is to use each pixel as parameter for the SVM. To make the train data as compact and accurate as possible, before taking the binary value all the images have to be resized to $30 \times 30$ pixels. Then, to make sure there will only 1 and 0 value for each pixel, image threshold should be done.

However, the bad side of using binary image is it is hard to differ from allowed sign to disallowed signs. On dealing with this problem, there will be 2 phases of SVM. First is to detect the meaning of the sign. Second is to classify it as allow or disallow. Other signs will be treated as allow signs. For more details about the use of SVM in traffic sign recognition can be found on Fleyeh's thesis. [7]

\section{EXPERIMENTS}

On the experiment, two main tests will be done. First is to test the detection algorithm and second is to test the recognition algorithm. Detection algorithm test successful parameter is to localize traffic sign with noise as minimum as possible, meanwhile recognition algorithm test successful parameter is to answer correctly the class of the detected traffic sign.

Detection algorithm consists of preprocessing image to binary image with color-based method threshold and 
approximating shape by using Ramer-Douglas-Peucker algorithm. The result of the detection algorithm will be localized traffic sign. Color-based method is used because of its speed and the simplicity of traffic sign. Traffic sign normally contains a lot of only one color. For instance, the experiment result on figure 5 shows a very good result of color threshold and ellipse detection on the approximated shape.

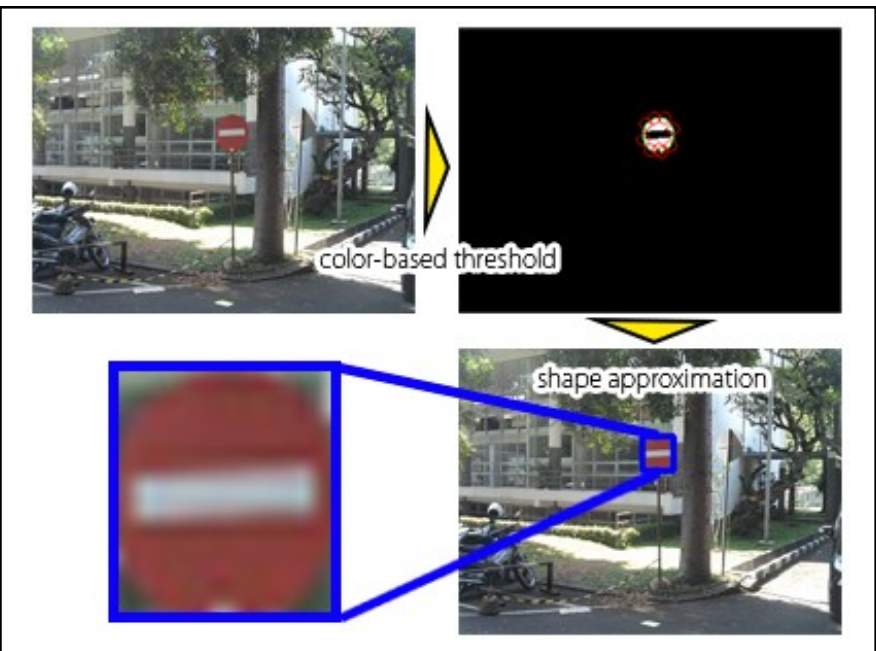

Figure 5. Color-based threshold and shape approximation on traffic sign detection successfully detect a forbidden sign.

However, color-based threshold has a drawback. In a not ideal environment such as too bright or too dark it will cause noises even though theoretically using CIELab color space + hue will make it light invariance. In the experiment using edited image with very high brightness, the algorithm gives many noises. Despite of many noises produced, the color-based threshold and shape approximation algorithm have successfully detected the traffic sign as shown in figure 6 . But, this is still a bad result; Better color-based threshold which produce less noises must be made in order to achieve more accurate traffic sign detection.

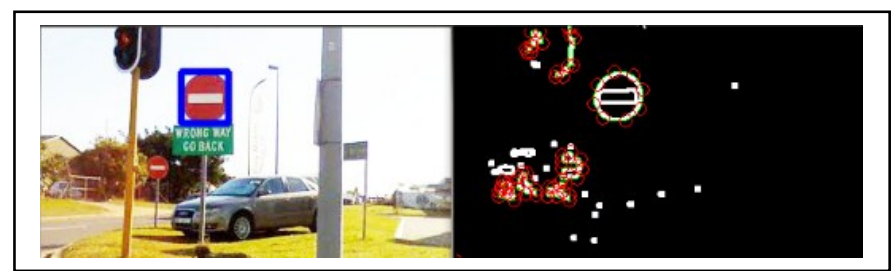

Figure 6. Too many noises produced on extreme brightness condition.

Other tests such as to test skew and multiple traffic signs are conducted too. As the theory said, the results on these cases are good (small traffic sign in figure 6 is not detected because it is too far). With color-based method and shape approximation, both problems can be handled. Figure 7(a) and 7(b) are the experiment results on skew and multiple traffic signs.

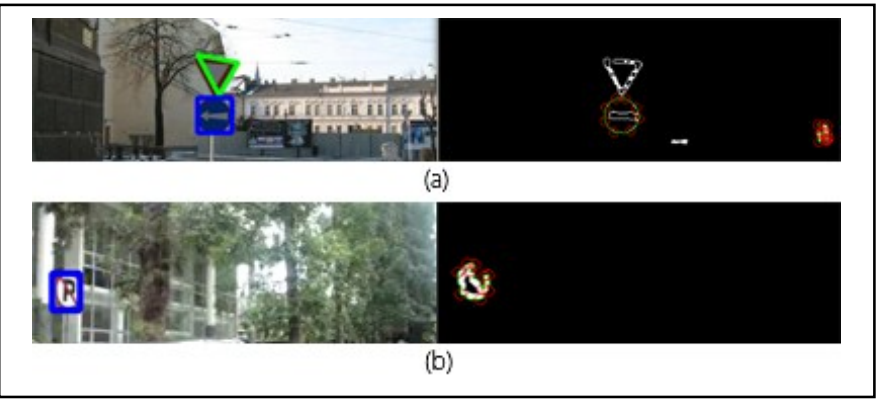

Figure 7. (a) multiple traffic signs (b) skewed traffic sign.

Also a test on the same image as the implemented IHLS method [6]. On this test, the method proposed produce the almost the same result without calculating the square-root and divide like in the reference. Figure 8 shows the almost same result

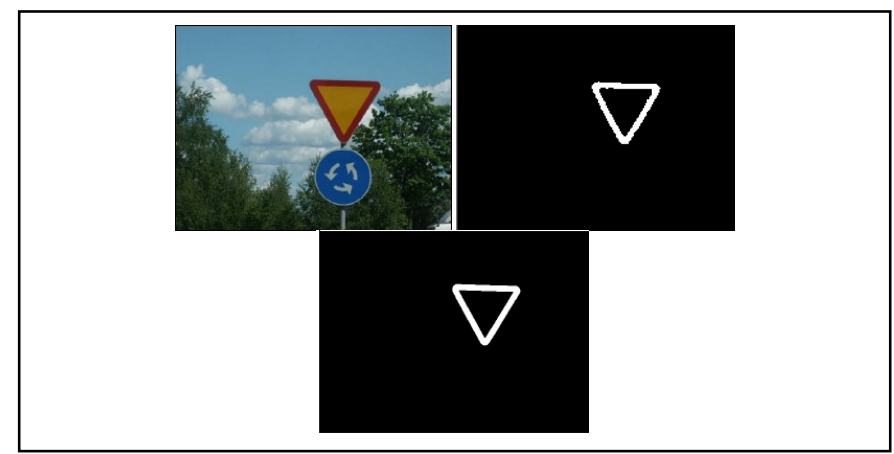

Figure 8. From upleft to downright: Original image, proposed method, IHLS method

Before advancing the experiment to the recognition step, there is one more test for the detection. It is the detachment of attached signs. By using closed object fitEllipse2 from OpenCV, the result on figure 9 is achieved. As we can see, there are actually three red road signs which are detected as one object. To detach it, we can use the shape-arc algorithm and the result will be on the figure shown in figure 10 .

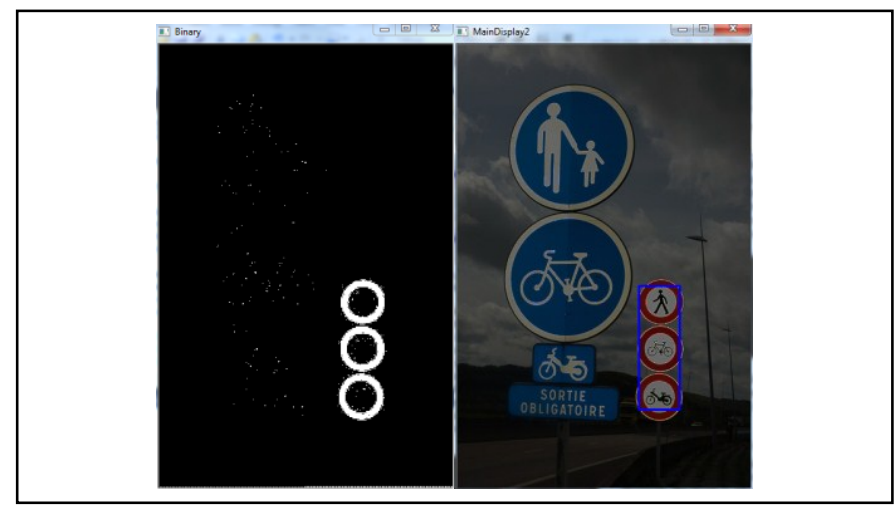

Figure 9. Three road signs detected as one because of attached issue 


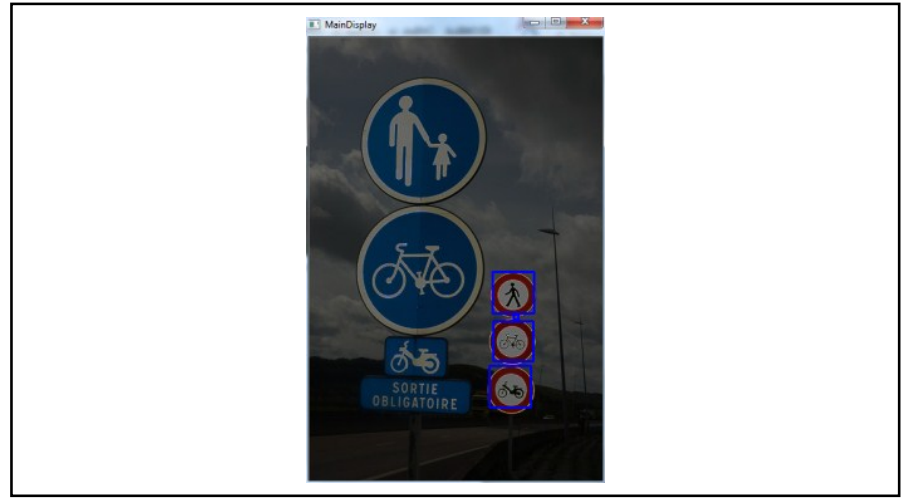

Figure 10. By using shape-arc algorithm three signs can be detected

Other test is conducted as a prove of improvement from Fleyeh's thesis as it was mentioned on the conclusion section of what can be improved. In figure 11, the same image used in Fleyeh's thesis is successfully detached.
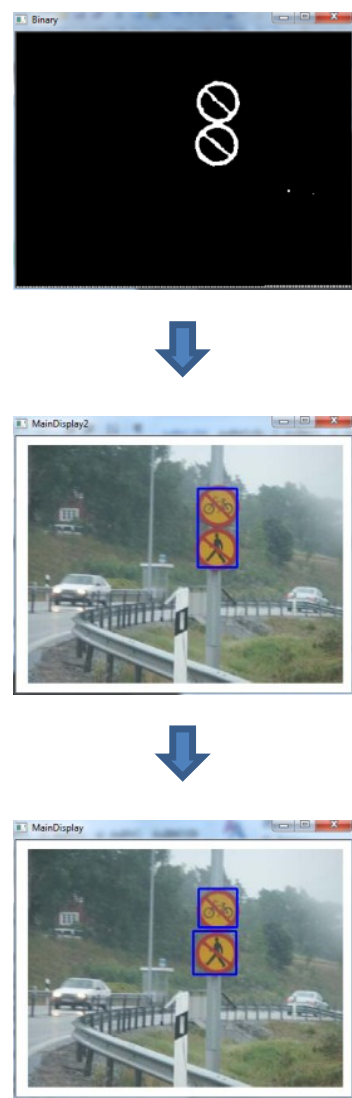

Figure 11. Detachment by using shape-arc algorithm

Final experiment, is to test the recognition accuracy. Before start the recognition, preprocess of detected images has to be done. All detected images have to be converted to a $30 \mathrm{x}$ 30 pixels image with binary value of 1 and 0 . To get the binary value, histogram equalization and threshold can be done. The result of the conversion will be like Table 1

TABLE I. CONVERSION RESULT FROM DETECTED SIGN

\begin{tabular}{|c|c|}
\hline Detected as a Sign & Conversion Result \\
\hline 9 & \\
\hline & \\
\hline & \\
\hline & \\
\hline
\end{tabular}

All of the binary image pixel will be the parameter on making the vector for the SVM. SVM used is C-SVM with linear kernel. After the SVM has been trained by the binary image, the final recognition step can be done. Table 2 shows the result of the test of detected signs and noises.

TABLE II. CLASSIFICATION RATES USING LINEAR KERNEL AND C-SVM TYPES WHEN BINARY IMAGES ARE USED.

\begin{tabular}{|l|l|l|l|}
\hline Class & Train images & Test images & Accuracy \\
\hline 6 & 77 & 100 & $94 \%$ \\
\hline 6 & 83 & 100 & $100 \%$ \\
\hline 7 & 85 & 100 & $100 \%$ \\
\hline 7 & 86 & 105 & $98.10 \%$ \\
\hline
\end{tabular}

TABLE III. CLASSIFICATION RATES OF SIGN RIMS AND SPEED-LIMIT SIGNS USING DIFFERENT KERNELS AND SVM TYPES WHEN BINARY IMAGES ARE USED. [7]

\begin{tabular}{|l|l|l|l|l|l|}
\hline \multirow{2}{*}{$\begin{array}{l}\text { SVM } \\
\text { Type }\end{array}$} & \multirow{2}{*}{ Kernel } & \multicolumn{2}{|l|}{ Sign Rims } & \multicolumn{2}{l|}{ Speed-Limits } \\
\cline { 3 - 6 } & & Training \% & Testing \% & Training \% & Testing \% \\
\hline C-SVM & Linear & 100 & 100 & 100 & 100 \\
\hline
\end{tabular}




\begin{tabular}{|l|l|l|l|l|l|}
\hline \multirow{2}{*}{ V -SVM } & Polynomial & 100 & 100 & 100 & 100 \\
\cline { 2 - 6 } & RBF & 100 & 100 & 100 & 100 \\
\cline { 2 - 6 } & Sigmoid & 100 & 100 & 100 & 98.7 \\
\cline { 2 - 6 } & Polynomial & 100 & 98.9 & 100 & 98.9 \\
\cline { 2 - 6 } & RBF & 100 & 97.8 & 100 & 98.7 \\
\cline { 2 - 6 } & Sigmoid & 100 & 98.9 & 100 & 98.7 \\
\hline
\end{tabular}

As a comparison, Table 3 shows the result of other kind of SVM conducted by Hasan Fleyeh on his thesis [7]. This is just one of the result, for more and complete result can be found on his thesis.

\section{DISCUSSION}

Using color-based method, shape-arc estimation and SVM for traffic sign recognition is very fast. It can be used for realtime traffic sign recognition. However, the result is still below expectation. To improve the result many things can be done. First is to add the training data for the SVM. With more relevant data the result will be more accurate too. As for the detection, a better threshold could be developed because threshold with only $1, a, b$ and hue is proved as not enough on eliminating all noises from the image. Also, computational cost difference between the proposed method and IHLS method which gives the best result has not been conducted. As on improving the detection algorithm, shape-arc algorithm can be used to detach an attached group of signs. However it is expensive and can only detect specific shapes. A more robust algorithm for the shape-arc algorithm can be developed on the future to detach glued traffic signs.

An improvement on the camera will also be significant. For example, the use of adaptive camera that can change the captured image to desired environment will give a lot improvement on make robust traffic sign recognition against the illumination factor. After all, color-based method and SVM is a fast algorithm for real-time traffic sign recognition but still need improvements on the accuracy.

\section{CONCLUSION}

Color filtering using CIELab color space + hue is efficient and produces good result, unlike its theory says it is illumination proof, the result shows illumination issue still occur for extreme condition. Shape-arc estimation is good on detaching attached signs but consumes a lot of computational cost on extracting the arcs. The use of binary image for SVM with linear kernel and C-SVM is very accurate on recognizing traffic signs. Experiment shows the average of $97 \%$ accuracy.

\section{ACKNOWLEDGMENT}

David Soendoro thanks Prof. Dr. Ir. Iping Supriana for his supervise on doing this paper as a part of David Soendoro's final project. He also thanks everyone that has given idea for him on doing this paper.

\section{REFERENCES}

[1] Lorsakul, A. and Suthakorn, J. "Traffic Sign Recognition Using Neural Network on OpenCV: Toward Intelligent Vehicle/Driver Assistance System" (PDF). Center for Biomedical and Robotics Technology (BART LAB), Department of Biomedical Engineering, Faculty of Engineering, Mahidol University, Thailand. 2005.

[2] Bahlmann, C. and friends. "A System for Traffic Sign Detection, Tracking, and Recognition Using Colour, Shape, and Motion Information" (PDF). Siemens Corporate Research, Inc. Princeton, USA. 2005.

[3] de la Escalera, A., Moreno, Luis E., Salichs, M.A., and Armingol, J.M.. "Road Traffic Sign Detection and Classification" (PDF). IEEE. 1997.

[4] de la Escalera, Armingol, J.M., Mata, M.. "Traffic Sign Recognition and Analysis for Intelligent Vehicles" (PDF). Division of Systems Engineering and Automation, Universidad Carlos III de Madrid, Madrid, Spain. 2002.

[5] Fairchild M. D.. "Color Appearance Models: CIECAM02 and Beyond". Slides from a tutorial at the IS\&T/SID 12th Color Imaging Conference. 9 November 2004. Retrieved 19 September 2007.

[6] Fleyeh H.. "Color Detection and Segmentation for Road and Traffic Signs”. Conference on Cybernetics and Intelligent Systems. Singapore, 1-3 December, 2004

[7] Fleyeh H.. "Traffic and Road Sign Recognition". Thesis of Hasan Fleyeh from Napier University for the degree of Doctor of Philosophy, July 2008

[8] Hershberger J. \& Snoeyink J., "Speeding Up the Douglas-Peucker LineSimplification Algorithm", Proc 5th Symp on Data Handling, 134-143 (1992). UBC Tech Report TR-92-07 available at http://www.cs.ubc.ca/cgi-bin/tr/1992/TR-92-07

[9] Schanda J. (2007). Colorimetry. Wiley-Interscience. p. 61. ISBN 9780470049044.

[10] WHO World Health Report 2002. Countries grouped by WHO Mortality Stratum, with Developing Countries representing regions with High and Very High Mortality, and Developed Countries representing regions with Low and Very Low Mortality.

[11] World Health Organization (2004). "Annex Table 2: Deaths by cause, sex and mortality stratum in WHO regions, estimates for 2002" (pdf). The world health report 2004 - changing history. Retrieved 2008-11-01. 